\title{
EARLY RENAL FAILURE AFTER MESALAZINE (CASE REPORT)
}

\author{
Dalibor Musil, Josef Tillich
}

II Internal Clinic University Hospital, Palacký University Olomouc, Czech Republic

Received July 15, 2000

Key words: 5-Aminosalicylic acid / Mesalazine / Renal failure / Tubuloinerstitial nephritis / Ulcerative colitis

\begin{abstract}
An early onset of asymptomatic renal failure in a young man with ulcerative colitis treated with high-dose mesalazine is the subject of this case report. After mesalazine withdrawal, renal function was quickly restored. In contrast with published case reports, the onset of renal impairment occurred very early. Serum urea reached pathological values 27 days and serum creatinine 15 days after mesalazine treatment was started. The diagnosis of renal impairment was supported by urine analysis (both semi-quantitative and quantitative) and dynamic renal isotope scanning.
\end{abstract}

5-Aminosalicylic acid (5-ASA) is the first line therapy for inflammatory bowel disease. It can be administered as sulfasalazine (5-ASA + sulfapyridine), mesalazine (5-ASA + resins) or olsalazine (5-ASA + 5-ASA). The recent trend prefers mesalazine and olsalazine to sulfasalazine due to their lesser frequent adverse effects but its potential myelotoxicity, hepatotoxicity and nephrotoxicity still must be considered ${ }^{2,3}$.

Nephrotoxicity is manifested as indolent interstitial nephritis that - if not diagnosed and treated - can progress to terminal renal failure even after preparation withdrawal. Nephrotoxicity must be suspected in any case of renal function impairment in a patient treated with 5-ASA ${ }^{2}$.

All published cases of renal failure due to mesalazine were caused by tubulointerstitial nephritis, usually confirmed with renal biopsy. There was no difference between number of patients with ulcerative colitis and Crohn disease. All the patients were young people under 34 years of age (20 to 34 years), more frequently men $(86 \%)$ than women (14\%). Patients used 1.2 to $3 \mathrm{~g}$ of mesalazine daily at least for 12 months (interval 12 months to 7 years). Previously reported cases describe chronic interstitial nephritis occuring after months to years of mesalazine use $e^{1,2,3,5,6,7,8,9}$. We report the case of a young man with asymptomatic renal failure development after three weeks administration of mesalazine.

\section{CASE DESCRIPTION}

23-year-old university student suffered for three years from diarrhea, sometimes with blood in stools, and from painful rectal discomfort. He visited a physician first time on November $9^{\text {th }} 1999$, few days after fever, abdominal pain, anorexia and weight loss had occured. So far he was not ill and did not take any drugs. Sigmoidoscopy showed proctocolitis of grade II.-III. $30 \mathrm{~cm}$ from anus, other parts of colon were not examined due to pain. The diagnosis of ulcerative colitis was confirmed by biopsy of mucosa. The out-patient treatment started with Asacol orally (mesalazine $2.4 \mathrm{~g}$ daily), Salofalk enemas (4g of mesalazine daily) and iron substitution (Aktiferrin comp. $2 \times 1$ tbl.)

Two weeks of home treatment with mesalazine was not succesful and on November $23^{\text {rd }}$ the patient was admitted to our hospital. The treatment continued with the oral administration of prednisolone $60 \mathrm{mg}$ daily, Salofalk (mesalazine $1.5 \mathrm{~g}$ daily), famotidine, iron, paracetamol and metronidazol. Since the $6^{\text {th }}$ day of hospitalization gentamicine had been administered for four days (240 mg in intramuscular injection, since November $29^{\text {th }}$ to December $2^{\text {nd }} 1999$ ). Hydrocortisone $300 \mathrm{mg}$ daily in saline intravenous infusion was added on December $3^{\text {rd }}$ for persisting bloody dirrhea, fever and abdominal pain and the mesalazine dose (Asacol) was raised to $3.2 \mathrm{~g}$ daily. Both parenteral (Nutriflex peri $2200 \mathrm{ml}$ daily) and enteral (Nutrison standard $1000 \mathrm{kcal}$ per day) nutritions were administered.

During hospitalization asymptomatic renal failure developed. Serum urea and creatinine rapidly increased, the highest level reached on December $7^{\text {th }} 1999$, after 29 days of mesalazine administration (urea $15.8 \mathrm{mmol} / \mathrm{l}$, creatinine $202 \mu \mathrm{mol} / \mathrm{l}$ - see Table 1$)$. Simultaneously isostenuria (urine specific weight 1010), proteinuria and leukocyturia occurred that persisted after mesalazine withdrawal (see Table 2).

The quantitative urine analysis corresponded: high leukocyturia (16 667 elem./min - normally 0-4000) and mild proteinuria. Erythrocytes 1667 elem./min. (normal), cylinders 0, specific weight 1010 . 
Serious immunologic abnormalities were not found: autoantibodies (ANF, ANCA, revmatoid factor) were negative, circulating immune complexes normal, complement C3 and C4 measurement slightly lower. Serum immunoelectrophoresis - non-significant increase in the all groups of immunoglobulines and higher increase in IgA: IgM $2.95 \mathrm{~g} / \mathrm{l}$ (normally under $2 \mathrm{~g} / \mathrm{l}$ ), IgA $6.65 \mathrm{~g} / \mathrm{l}$ (normal range 1.5-4.0), IgG $20 \mathrm{~g} / \mathrm{l}$ (normal range under $15 \mathrm{~g} / \mathrm{l}), \mathrm{IgE} 173 \mathrm{~g} / \mathrm{l}$ (normal under $150 \mathrm{~g} / \mathrm{l})$. Serum proteinase 3 and myeloperoxidase were negative.

The functional renal examination: decreased glomerular filtration rate $(0.73 \mathrm{ml} / \mathrm{s})$ and tubular resorption (0.974). Repeated urine cultures brought negative results. The ultrasound: normal ultrasonographic image of both kidneys. The patient's diuresis at the begining was normal, later during restitution of the renal function polyuria appeared (see Table 2).

During the period of maximal renal function impairment the patient underwent dynamic renal isotope scanning. The excretion started in time but it was considerably slowed down. Kidneys were normally shaped, radioisotope transport slowed down, relatively more at the left side (left kidney excretion half-time 148 minutes, maximal transit time 810 seconds, right kidney excretion half-time 50 minutes, maximal transit time 290 seconds). Kidney biopsy was not carried out.

Since the patient had never been treated for any kidney disease or diabetes mellitus, had not any symptoms or clinical signs of previous renal impairment, had been well-hydrated and without fever and all other investigations had not explicitely shown bilateral renal parenchyma impairment, we considered a role of the mesalazine nephrotoxic effect. This effect was consecutively confirmed by rapid normalization of both renal function and urine findings after mesalazine withdrawal.

On December $8^{\text {th }} 1999$ Asacol was withdrawn and the ulcerative colitis treatment continued only with corticosteroids. During a short time period serum urea and creatinine decreased to normal levels, glomerular filtration rate increased partially (December $20^{\text {th }} 1999$ : $1.26 \mathrm{ml} / \mathrm{sec}$, normally $2.19 \mathrm{ml} / \mathrm{s}$, i.e. minus $38 \%$ ) and proteinuria disappeared. Polyuria, isostenuria and leukocyturia persisted (see Table 2). The patient was discharged after 18 days of hospitalization was in good condition, without fever or colitic symptoms, treated only with prednisolone ( $40 \mathrm{mg}$ daily).

4 and 8 weeks after discharge is the patient without problems. Laboratory findings after 8 weeks showed neither signs of the ulcerative colitis activity nor renal impairment (negative chemical and microscopic urine analysis, urine specific weight 1023 , urea $4.1 \mathrm{mmol} / \mathrm{l}$, creatinine $82 \mu \mathrm{mol} / \mathrm{l})$.

\section{DISCUSSION}

The recent trend in the therapy of IBD prefers 5-ASA preparations, especially mesalazine, to sulfasalazine for frequent adverse effects, linked usually to contained sulfapyridine. However, mesalazine treatment has its risks - and tubulointerstitial nephritis as obviously most serious of them. Laboratory tests in rats have proved the nephrotoxic effect of mesalazine after its short-time intravenous application ${ }^{12}$.

5-ASA is released in the colon, after the resin coat dissolution of the mesalazine tablet. Then about one fifth of mesalazine is absorbed and later excreted by kidneys. Tubular and papillary necrosis after 5-ASA had been proved in the early $1970 \mathbf{s}^{12}$, cases of nephrotic syndrome both after sulfasalazine ${ }^{11}$ and mesalazine ${ }^{10}$ were described in the $1980 \mathrm{~s}$. Nephrotoxicity was most frequently described with the preparation Asacol - the reason can be that the preparation has been sold for the longest time $2,3,6$.

The 5-ASA structure is similar to that of salicylates and phenacetine and 5-ASA can cause nefropathy like the non-steroid antiinflammatory drugs (NSAIDs) - and the nephropathy reminds one of analgetic nephropathy characterized by interstitial nephritis and papillary necrosis $^{6,9}$. The comparison of acetylsalicylic acid (ASA) and its derivates (3-, 4- and 5-Aminosalicylic acids) cytotoxicity on in vitro cell cultures have not been found to have any characteristic nephrotoxic effect. The explanation of tubulointerstitial nephritis after 5-ASA has not been found yet ${ }^{4}$.

104 cases of renal impairment or pathological urinary tests connected with the mesalazine treatment have been reported during the past 13 years (1985-1998) in Great Britain (Committee on Safety of Medicines). 35 cases $(33.7 \%)$ involved interstitial nephritis ${ }^{2}$.

Exact incidence of the complication is not known but it is estimated to be about one percent of patients treated with mesalazine and cases of clinically important interstitial nephritis are less than one in 500 patients treated with mesalazine ${ }^{2}$.

Bioptic samples mentioned in literature showed both glomerular and tubular damage, usually with plasmocytic and eosinophilic infiltrates ${ }^{1,2,9}$.

Severe chronic and progressive interstitial nephritis is the most frequent clinical form, that appears after few months of treatment - all described cases involve the mesalazine administration for at least 12 months, the diagnosis was made even after 7 years of the continual treatment in a case 7 . The early signs were sterile pyuria and the low urine specific weight, as in salicylates ${ }^{6}$.

Usually, the renal impairment was asymptomatic, unveiled by regular follow up during hospitalization, for the relapse of IBD ${ }^{1,2,3,6}$. Only in one of the cases ${ }^{5}$ there were six months of weight loss, fatigue and dyspnea and another case showed "foamed" urine, which was noticed by the patient ${ }^{2}$.

If the mesalazine nephrotoxicity is diagnosed earlier than 10 months after the treatment started, the renal 
function restoration after withdrawal is observed in $85 \%$ of patients. Only the partial improvement of renal function can be expected in one third of patients after 18 months of the treatment ${ }^{2}$. The prognosis is uncertain ${ }^{6}$.

The precise mechanism of impairment is not clear, but most probably it is a type IV hypersensitivity reaction $^{2}$.

Renal failure in our patient, contrary to cases published so far, has developed very early. The serum urea level reached to a pathological value after 27 days. Also the creatinine level rose 15 days after the mesalazine administration started and progresses rapidly.

Gentamicine influence at renal function can be passed - the therapy started later and lasted only for 4 days. Also the influence of prerenal mechanism (dehydration) can be excluded - the patient has been wellhydrated all the time.

The rapid progress of renal failure as described here is quite unusual but other features are characteristic - a young man with high doses of mesalazine and asymptomatic renal failure, was retreated after mesalazine withdrawal.

Mechanisms of renal impairment can be only supposed - some features (allergy in patient's history, higher serum $\mathrm{IgE}$ and a quick onset and regression of renal damage) could lead to thoughts about the first type hypersensitivity reaction but other characteristic signs and symptoms were missing (rash, urtica or eosinophilia).

\section{CONCLUSION}

The case of asymptomatic renal failure with an early onset in a young man with ulcerative colitis treated with high-dosed mesalazine is the subject of this case report. Quick and complete restoration of renal function succeeded after mesalazine withdrawal. The 5-ASA nephrotoxicity must be considered in any patient treated with this drug. Serum urea and creatinine should be checked monthly for the first three months, 3-monthly for the remainder of the first year and annualy thereafter and in every relapse of IBD or clinical signs of renal impairment.

The 5-ASA therapy in a patient with known history of its nephrotoxicity should be stopped, instead the patient should be treated with a long-time treatment using prednisolone or prednisolone+azathioprine $\mathrm{e}^{1,2,3,11}$.
Table 1. Asymptomatic renal failure

\begin{tabular}{|c|c|c|c|}
\hline $\begin{array}{c}\text { Date } \\
\text { (days of mesalaxine } \\
\text { administration) }\end{array}$ & $\begin{array}{l}24.11 .99 \\
\text { (15 days) }\end{array}$ & $\begin{array}{c}7.12 .99 \\
\text { (29 days) }\end{array}$ & 17.12.99 \\
\hline Urea (mmol/h) & 4.6 & 15.8 & 6.0 \\
\hline Creatinine $(\mu \mathrm{mol} / \Omega)$ & 126.2 & 202.0 & 99.0 \\
\hline
\end{tabular}

Table 2. Urine analysis

\begin{tabular}{|c|c|c|c|c|}
\hline & 24.11 .99 & 7.12 .99 & 9.12 .99 & 13.12 .99 \\
\hline Protein & 1 & 3 & 1 & 0 \\
\hline Leucocytes & $40-50$ & $10-15$ & 4 & 10 \\
\hline Erythrocytes & $0-4$ & 1 & 0 & 0 \\
\hline $\begin{array}{c}\text { Spec. Weight } \\
\text { (kg/m) }\end{array}$ & 1010 & 1010 & 1010 & 1010 \\
\hline $\begin{array}{c}\text { Diuresis } \\
\text { (m/day) }\end{array}$ & 1500 & 2500 & 3000 & 3000 \\
\hline
\end{tabular}

\section{REFERENCES}

1. Brouillard, M., Katz, K. O., Henning, N. P., Reimann, D. S., Myers, B. (1998): Chronic interstitial nephritis and mesalazine: 3 new cases? Gastroenterol. Clin. Biol., 22, 724-726.

2. Popoola, J., Muller, A. F., Pollock, L., O’Donnell, P., Carmichael, P., Stevens, P. (1998): Late onset interstitial nephritis associated with mesalazine treatment. BMJ, 317, 795-797.

3. Calvino, J., (1998): Mesalazine-associated tubulo-interstitial nephritis in inflammatory bowel disease. Clin. Nephrol., 49, 265-267.

4. Noble, E., Janssen, L., Dierick, P. J. (1997): Comparative cytotoxicity of 5-aminosalicylic acid (mesalazine) and related compounds in different cell lines. Cell. Biol. Toxicol., 13, 445-451.

5. Hamling, J., Raedler, A., Helmchen, U., Schreiber, S. (1997): 5-Aminosalicylic acid-associated renal tubular acidosis with decreased renal function in Crohn's disease. Digestion, 58, 304-307.

6. World, M. J., Stevens, P. E., Ashton, M. A., Rainford, D. J. (1996): Mesalazine-associated interstitial nephritis. Nephrol. Dial. Transplant., 11, 614-621.

7. Garcia-Diaz, M., (1995): Acute renal failure associated with 5-aminosalicylic acid in inflammatory bowel disease. Gastroenterol. Hepatol., 18, 18-21.

8. Smilde, T. J., van Liebergen, F. J., Koolen, M. I., Gerlag, P. G., Assman, K. J., Berolen, J. H. (1994): Tubulointerstitial nephritis caused by mesalazine (5-ASA) agents. Ned. Tijdschr. Geneeskd., 138, 2557-2561.

9. Thuluvath, P. J., Ninkovic, M., Calam, J., Anderson, M. (1994): Mesalazine induced intersticial nephritis. Gut, 35, 1493-1496.

10. Novis, B. H., Korzets, Z., Chen, P., Bernheim, J. (1988): Nephrotic syndrome after treatment with 5-aminosalicylic acid. BMJ, 296, 1442.

11. Barbour, V. M., Williams, P. F. (1990): Nephrotic syndrome associated with sulphasalazine. BMJ, 301, 818.

12. Calder, I. C., Funder, C. C., Green, C. R., Ham, K. N., Tange, J. D. (1972): Nephrotoxic lesions from 5-aminosalicylic acid. Br. Med. J., 1, 152-154. 